\title{
CUBIC EXTENSIONS OF FLAG-TRANSITIVE PLANES, I. EVEN ORDER
}

\author{
YUTAKA HIRAMINE, VIKRAM JHA, and NORMAN L. JOHNSON \\ (Received 7 September 1999)
}

\begin{abstract}
The collineation groups of even order translation planes which are cubic extensions of flag-transitive planes are determined.

2000 Mathematics Subject Classification. Primary 51E.
\end{abstract}

1. Introduction. In this article, we begin the analysis of translation planes of order $q^{3}$ that admit collineation groups $G$ which leave invariant a subplane $\pi_{0}$ of order $q$, act flag transitively on $\pi_{0}$ and act transitively on the set of components not in $\pi_{0}$.

In two previous, related articles (see [6, 7]), the general study of translation planes which are extensions of flag-transitive planes is undertaken.

An "extension of a flag-transitive plane" is an affine plane $\pi$ containing an affine subplane $\pi_{0}$ and a collineation group which leaves $\pi_{0}$ invariant, acts flag-transitively on $\pi_{0}$ and acts transitively on the parallel classes of $\pi$ not in $\pi_{0}$.

The main results of these two articles are as follows.

THEOREM 1.1 (see Hiramine et al. [7]). Let $\pi$ be a finite translation plane which is a quadratic extension of a flag-transitive plane $\pi_{0}$.

Then $\pi$ is either Desarguesian, Hall, or the derived likeable Walker plane of order 25.

In particular,

(1) if the associated collineation group is non-solvable, then $\pi$ is Desarguesian and

(2) if the associated collineation group is solvable, then $\pi$ is Hall, Desarguesian of order 4 or 9 or the derived likeable Walker plane of order 25.

The motivation to consider "cubic extensions" arises partially from the following result.

THEOREM 1.2 (see Hiramine et al. [6]). Let $\pi$ be a finite translation plane of order $q^{n}$ which is a solvable extension of a proper flag-transitive plane $\pi_{0}$ of order $q$. Let $G$ denote the corresponding group.

Then one of the following occur.

(1) $\pi$ is Desarguesian and $(q, n)$ is in $\{(2,2),(2,3),(3,2),(3,3),(2,5)\}$.

(a) For $(2,2),(2,3)$, the group $\mathrm{SL}(2,2)$ is a $(3,2)$ - or $(3,6)$-transitive group, respectively.

(b) For $(3,2),(3,3)$, the group $\mathrm{SL}(2,3)$ is a $(4,6)$ - or $(4,24)$-transitive group, respectively.

(c) For $(2,5)$, the group $\mathrm{SL}(2,2) \times Z_{5}$ is a $(3,30)$-transitive group. 
(2) $n=2$ and $\pi$ is either
(a) Hall or
(b) the derived likeable Walker plane of order 25.

(3) $n=3$.

(4) $n>3$ and $q=2,3$, or 4 .

Furthermore, one of the following occurs.

(a) $q=2$ and there is a normal subgroup generated by elations isomorphic to $\operatorname{SL}(2,2)$ which acts doubly-transitively on the infinite points of $\pi_{0}$. Also, the Sylow 2-subgroups have order 2 and the full group $G_{\left[\pi_{0}\right]}$ which fixes $\pi_{0}$ pointwise has index 6 so that $\mathrm{SL}(2,2) G_{\left[\pi_{0}\right]}$ is the full translation complement.

In addition, if $n$ is even then the spread is a union of Desarguesian nets of degree 5 containing $\pi_{0}$ and there is a regular partial 2-parallelism of $2^{n-1}-12$-spreads in PG $(2 n-1,2)$,

(b) $q=3$ and $n$ is even. Furthermore, there is a normal subgroup generated by 3elements such that the restriction to $\pi_{0}$ is isomorphic to $\operatorname{SL}(2,3)$ and which acts doubly transitively on the infinite points of $\pi_{0}$. The Sylow 3-subgroups are non-planar groups of order 3 and the full group $G_{\left[\pi_{0}\right]}$ which fixes $\pi_{0}$ pointwise has index $24 \operatorname{soSL}(2,3) G_{\left[\pi_{0}\right]}$ is the full translation complement.

If the 3-elements elements are elations, the spread is a union of Desarguesian nets of degree 10 containing $\pi_{0}$ and there is a regular partial 2-parallelism of $\left(3^{n-1}-1\right) / 2$ 2 -spreads in PG(2n-1,3). Furthermore, if the 3-elements are not elations then $n \geq 20$.

(c) $q=4$ and $n=4$.

(d) $q=4$ and $n>4$. Then all involutions are elations and there is a normal subgroup generated by elations that acts doubly transitively on the infinite points of $\pi_{0}$.

Furthermore, the Sylow 2-subgroups are cyclic of order 4 and there is a normal 2complement. If $\tau$ is a collineation of order 4 then $\pi$ may be decomposed into a direct sum of $n$ cyclic $\tau \mathrm{GF}(2)$-submodules of dimension 4 and each Sylow 2-group pointwise fixed subspace has cardinality $2^{n}$.

There are very strong reasons why the cubic extensions do not appear in the statement of the previous result.

In Jha et al. (see $[8,9]$ ), a classification is given of a large subclass of translation planes called generalized Desarguesian planes of order $q^{3}$ that admit GL $(2, q)$. There are many mutually nonisomorphic planes of this type and where the kernel of the plane may be chosen in a variety of ways.

In these planes, the associated vector space is a standard GF $(q) \mathrm{GL}(2, q)$ module. The effect of this is that a group isomorphic to $\operatorname{SL}(2, q)$ is generated by elation groups and that $\mathrm{GL}(2, q)$ leaves invariant each subplane of order $q$ incident with the zero vector in the associated $\operatorname{GF}(q)$-regulus net defined by the elation axes of $\operatorname{SL}(2, q)$. Furthermore, there are always infinite orbits of lengths $q+1$ and $q^{3}-q$. In a translation plane, there is always a translation subgroup acting transitive on any affine subplane so we obtain a tremendous variety of cubic extensions of a Desarguesian flag-transitive plane admitting non-solvable collineation groups when $q>3$.

In this article, we are also able to note that the Lüneburg-Tits plane of order $2^{18}$ is a cubic extension of a Lüneburg-Tits subplane of order $2^{6}$. 
We divide the consideration of cubic extensions into planes of even and odd orders and develop here results for the even order case only.

Fundamentally, our results are mainly group theoretic.

We analyze the collineation groups of cubic extensions and are able to generally formulate a classification.

In particular, when the order is even then, without any further assumptions, we are able to show that there is always a collineation group isomorphic to either $\operatorname{SL}(2, q)$ or $S_{z}(\sqrt{q})$ which is generated by elation groups.

For convenience, we recall some definitions.

DEFINITION 1.3. If an affine plane $\pi$ of order $q^{n}$ admits a collineation group $G$ which has infinite point orbits of lengths $q+1$ and $\left(q^{n}-q\right)$, we call $\pi$ a " $\left(q+1, q^{n}-q\right)$ transitive plane" and $G$ a " $\left(q+1, q^{n}-q\right)$-transitive group."

If $G$ leaves a subplane $\pi_{0}$ of order $q$ invariant within the net of length $q+1$ and there is a collineation group transitive on the sets of affine and infinite points of $\pi_{0}$ and the infinite points of $\pi-\pi_{0}$ then $\pi_{0}$ is a flag-transitive affine plane and we shall call $\pi$ an "extension of $\pi_{0}$. ."

If the group of an extension is solvable, we shall call the plane a "solvable extension."

2. The Lüneburg-Tits planes. We have noted that there are a variety of translation planes of orders $q^{3}$ admitting a collineation group isomorphic to $\operatorname{SL}(2, q)$ which are cubic extensions of a Desarguesian affine plane of order $q$. We show that it is possible to have extensions of non-Desarguesian planes of order $q$. We first note a result on the structure of nets containing sufficiently many subplanes.

THEOREM 2.1. Let $\pi$ be a translation plane of order $q^{n}$ admitting a subplane $\pi_{0}$ of order $q$ and kernel $D$ isomorphic to $\mathrm{GF}\left(p^{t_{0}}\right)$ where $q=p^{r}$. Let $\mathcal{N}$ denote the net of degree $q+1$ determined by the components of $\pi_{0}$.

If there exist $n+1$ subplanes of $\mathcal{N}$ such that any $n$ of them direct sum to $\pi$, then all subplanes are isomorphic and there are exactly $\left(p^{t_{0} n}-1\right) /\left(p^{t_{0}}-1\right)$ subplanes of $\mathcal{N}$ incident with the zero vector.

Proof. Let $\mathscr{E}$ denote the enveloping algebra of $\mathcal{N}$ (the algebra generated by the slope mappings). Then all subplanes of $\mathcal{N}$ are irreducible $\mathscr{E}$-modules. Let the $n+1$ subplanes be denoted by $\pi_{i}$ for $i=1,2, \ldots, n+1$. Then $\bigoplus_{i=1}^{n} \pi_{i}=\bigoplus_{i=2}^{n+1} \pi_{i}$. Let $v_{1}=$ $v_{2}+\cdots+v_{n}+v_{n+1}$, where $v_{i} \in \pi_{i}$ for $i=1,2, \ldots, n+1$. Thus, $v_{n+1} \neq 0$ if and only if $v_{1} \neq 0$. Since the subplanes are $\mathscr{E}$-irreducible, the mapping $v_{1} \mapsto v_{n+1}$ is an $\mathscr{E}$ isomorphism. Similarly, we may choose any subplane $\pi_{j}$ and find an $\mathscr{E}$-isomorphism from $\pi_{j}$ onto $\pi_{n+1}$. Hence, all subplanes $\pi_{i}$ are $\mathscr{E}$-isomorphic to $\pi_{n+1}$. It then follows that $\mathscr{E}$ acts faithfully on $\pi_{1}$ so by Liebler [11] Theorem 1.4(b), the result follows.

In this section, we consider whether there are Lüneburg-Tits planes of order $q^{3}$ which are cubic extensions of a flag-transitive plane $\pi_{0}$. The subplane $\pi_{0}$ is Desarguesian or Lüneburg-Tits. In order to better consider the action of the collineation group, we develop some background on these planes and their representation. 
Proposition 2.2. Let $\pi$ be a Lüneburg-Tits plane of order $2^{2(2 r+1)}$ with spread in $\mathrm{PG}\left(3, K \simeq \mathrm{GF}\left(2^{2 r+1}\right)\right)$. Denote the points of $\pi$ by $\left(x_{1}, x_{2}, y_{1}, y_{2}\right)$ for all $x_{i}, y_{i} \in K, i=$ 1,2 . Let $x=\left(x_{1}, x_{2}\right), y=\left(y_{1}, y_{2}\right)$. Let $\sigma: x_{1} \mapsto x_{1}^{2^{r+1}}$ so that $x_{1}^{\sigma^{2}}=x_{1}^{2}$ for all $x_{1} \in K$.

Then the spread has the following representation:

$$
\{(x=0)\} \cup\left\{y=x\left[\begin{array}{cc}
b^{\sigma} & b+a^{\sigma+1} \\
b+a^{\sigma+1} & a^{\sigma}
\end{array}\right] ; a, b \in K\right\} .
$$

Proof. This representation may be obtained from that described in Lüneburg [12, Section 13] by the basis change:

$$
\left(z_{1}, z_{2}, w_{1}, w_{2}\right) \longmapsto\left(z_{1}, w_{1}, z_{2}, w_{2}\right) .
$$

Proposition 2.3. Let $\pi$ be a Lüneburg-Tits plane with representation as in the previous proposition. Then, the following mappings define collineations of $\pi$ :

$$
\begin{gathered}
\omega:(x, y) \longmapsto(y, x), \\
\left\{\tau(a, b):(x, y) \longmapsto(x, y) T_{a, b} ; a, b \in K\right\},
\end{gathered}
$$

where

$$
\begin{gathered}
T_{a, b}=\left[\begin{array}{cccc}
1 & a & a b+a^{\sigma+2}+b^{\sigma} & b \\
0 & 1 & a^{\sigma+1}+b & a^{\sigma} \\
0 & 0 & 1 & 0 \\
0 & 0 & a & 1
\end{array}\right], \\
\left\{\eta(k):(x, y) \longmapsto(x, y) M_{k} ; k \in K-\{0\}\right\},
\end{gathered}
$$

where

$$
\begin{gathered}
M_{k}=\left[\begin{array}{cccc}
1 & 0 & 0 & 0 \\
0 & k^{\sigma+1} & 0 & 0 \\
0 & 0 & k^{\sigma+2} & 0 \\
0 & 0 & 0 & k
\end{array}\right], \\
\left\{\operatorname{aut}\left(\rho_{z}\right):(x, y) \longmapsto\left(x^{\rho_{z}}, y^{\rho_{z}}\right) ; \rho_{z} \in \operatorname{Aut} K\right\},
\end{gathered}
$$

where

$$
\begin{gathered}
x^{\rho_{z}}=\left(x_{1}^{2^{z}}, x_{2}^{2^{z}}\right), \\
\left\{s(\alpha):(x, y) \longmapsto(x, y) K_{\alpha} ; \alpha \in K-\{0\}\right\},
\end{gathered}
$$

where

$$
K_{\alpha}=\left[\begin{array}{cccc}
\alpha & 0 & 0 & 0 \\
0 & \alpha & 0 & 0 \\
0 & 0 & \alpha & 0 \\
0 & 0 & 0 & \alpha
\end{array}\right] .
$$

Furthermore, the full translation complement is

$$
\left\langle\omega, \tau(a, b), \eta(k) \text {, aut }\left(\rho_{z}\right), s(\alpha)\right\rangle \quad \forall a, b \in K, \forall k, \alpha \in K-\{0\}, \text { and } \forall \rho_{z} \in \text { Aut } K \text {. (2.8) }
$$


In particular, the group

$$
\langle\omega, \tau(a, b), \eta(k)\rangle \simeq S_{z}(q)
$$

acts 2-transitively on the components of $\pi$.

Proof. This follows immediately from Lüneburg [12, Section 13] and, in particular (13.7), with the basis change indicated above. Also, see Section 1 of [12] where the mappings generating the Suzuki group $S_{z}(q)$ are considered. We note that although the notation used here is the same as that used by Lüneburg, our mappings reflect the basis change.

It is generally known that the Lüneburg-Tits spreads are regulus-free and this is easily verified using our matrix representation.

COROLLARY 2.4. The spreads for the Lüneburg-Tits planes are regulus-free.

Proof. Since the group acts 2-transitive on the components, we may assume that two of the components for a regulus are $x=0$ and $y=0$. Assume that a third component is

$$
y=x\left[\begin{array}{cc}
b_{0}^{\sigma} & b_{0}+a_{0}^{\sigma+1} \\
b_{0}+a_{0}^{\sigma+1} & a_{0}^{\sigma}
\end{array}\right]
$$

for a fixed $b$ and $a$ in $K$. Choose a new basis for the spread by applying the mapping

$$
(x, y) \longmapsto(x, y)\left[\begin{array}{cc}
b_{0}^{\sigma} & b_{0}+a_{0}^{\sigma+1} \\
b_{0}+a_{0}^{\sigma+1} & a_{0}^{\sigma}
\end{array}\right]^{-1} .
$$

If $x=0, y=0, y=x$ are components for a regulus $\mathscr{R}$ then it is well known that the remaining components have the general form

$$
y=x\left[\begin{array}{ll}
u & 0 \\
0 & u
\end{array}\right] \quad \forall u \in K-\{0\} .
$$

Thus, it must be that we have components of the form

$$
y=x\left[\begin{array}{cc}
u & 0 \\
0 & u
\end{array}\right]\left[\begin{array}{cc}
b_{0}^{\sigma} & b_{0}+a_{0}^{\sigma+1} \\
b_{0}+a_{0}^{\sigma+1} & a_{0}^{\sigma}
\end{array}\right] \quad \forall u \in K
$$

under the original representation. Hence, it follows that there must be elements in $K$ satisfying the following conditions: let $u b_{0}^{\sigma}=b_{1}^{\sigma}$ and $u a_{0}^{\sigma}=a_{1}^{\sigma}$ so that

$$
b_{0}+a_{0}^{\sigma+1}=b_{1}+a_{1}^{\sigma+1}
$$

So, we obtain

$$
b_{0}+a_{0}^{\sigma+1}=u^{\sigma^{-1}} b_{0}+u a_{0}^{\sigma} u^{\sigma^{-1}} a_{0}=u^{\sigma^{-1}}\left(b_{0}+a_{0}^{\sigma+1}\right) \quad \forall u .
$$

Thus,

$$
b_{0}=a_{0}^{\sigma+1} .
$$


Hence, we must have components of the form

$$
y=x\left[\begin{array}{cc}
u & 0 \\
0 & u
\end{array}\right]\left[\begin{array}{cc}
a_{0}^{\sigma(\sigma+1)} & 0 \\
0 & a_{0}^{\sigma}
\end{array}\right] \quad \forall u \in K
$$

However, we have elements of the form

$$
y=x\left[\begin{array}{cc}
a^{\sigma(\sigma+1)} & 0 \\
0 & a^{\sigma}
\end{array}\right] \quad \forall u \in K .
$$

Thus, if $v^{\sigma}=u$ then we obtain

$$
v^{\sigma(\sigma+1)}=v^{\sigma} \quad \forall v \in K
$$

which obviously is a contradiction.

THEOREM 2.5. Let $\pi$ be a Lüneburg-Tits plane of order $2^{2(2 r+1)}$.

(1) Then the spread for $\pi$ is a union of Desarguesian partial spreads of degree 5 that share a line.

Hence, there are Desarguesian subplanes $\pi_{0}$ of order 4 .

(2) If $2 r_{1}+1$ divides $2 r+1$ and $2 r_{1}+1>1$ then there is a Lüneburg-Tits subplane $\pi_{r_{1}}$ of order $2^{2\left(2 r_{1}+1\right)}$.

(3) Let $G$ denote the full translation complement of $\pi$. Let $K_{r_{1}}$ denote the subfield of $K$ isomorphic to $\mathrm{GF}\left(2^{2 r_{1}+1}\right)$. The stabilizer of $\pi_{r_{1}}, G_{\pi_{r_{1}}}$, is

$$
\left\langle\omega, \tau(a, b), \eta(k) \text {, aut }\left(\rho_{z}\right), s(\alpha)\right\rangle \quad \forall a, b \in K_{r_{1}}, \forall k, \alpha \in K_{r_{1}}-\{0\} \text {, and } \forall \rho_{z} \in \operatorname{Aut} K \text {. }
$$

(4) Given any subplane $\pi_{r_{1}}$ and $r_{1}>0$, there exist exactly $\left(2^{2 r+1}-1\right) /\left(2^{2 r_{1}+1}-1\right)$ Lüneburg-Tits subplanes that share the same components as $\pi_{r_{1}}$.

Proof. Restrict the points $\left(x_{1}, x_{2}, y_{1}, y_{2}\right)$ of $\pi_{r_{1}}$ so that $x_{i}, y_{i} \in K_{r_{1}}$. Then

$$
\{(x=0)\} \cup\left\{y=x\left[\begin{array}{cc}
b^{\sigma} & b+a^{\sigma+1} \\
b+a^{\sigma+1} & a^{\sigma}
\end{array}\right] ; a, b \in K_{r_{1}}\right\}
$$

is the set of components of $\pi_{r_{1}}$. The stabilizer subgroup is clearly as claimed as the collineations induced from automorphisms of $K$ leave $K_{r_{1}}$ invariant. Note that when $K_{0}$ is isomorphic to $\mathrm{GF}(2)$, it is immediate that the partial spread above for all $a, b$ in $K_{0}$ is a partial spread defined by a field of matrices isomorphic to GF(4). Hence, we obtain a Desarguesian partial spread of degree 5 .

When $r_{1}>0$, the group of kernel homologies of $\pi_{r_{1}}$ is isomorphic to $K_{r_{1}}-\{0\}$ so there exist $\left(2^{2 r+1}-1\right) /\left(2^{2 r_{1}+1}-1\right)$ images of $\pi_{r_{1}}$ under the kernel homology group and hence this number of subplanes isomorphic to $\pi_{r_{1}}$ sharing the components of $\pi_{r_{1}}$. Using the notation of Theorem 2.1, we have $q=2^{2\left(2 r_{1}+1\right)}$ so that $n=(2 r+1) /\left(2 r_{1}+1\right)$ and $p^{t_{0}}=2^{2 r_{1}+1}$. The maximum number of subplanes according to the result is $\left(p^{t_{0} n_{-}}\right.$ $1) /\left(p^{t_{0}}-1\right)=\left(2^{2 r+1}-1\right) /\left(2^{2 r_{1}+1}-1\right)$ so we have the proof of the result. 
We now consider if there are Lüneburg-Tits planes which are $n$-dimensional extensions of either a Desarguesian or a Lüneburg-Tits subplane.

THEOREM 2.6. A Lüneburg-Tits plane of order $h^{2 n}$ is an $n$-dimensional extension of a subplane of order $h^{2}$ if and only if one of the following occur:

(1) $h=2$ and $n=3$ or

(2) $h=2^{3}$ and $n=3$.

(3) Hence, there is a "chain" of 3-dimensional extensions $\pi_{0} \subseteq \pi_{1} \subseteq \pi$, where $\pi_{0}$ is Desarguesian of order $2^{2}, \pi_{1}$ is a Lüneburg-Tits plane of order $2^{6}$ and $\pi$ is a LüneburgTits plane of order $2^{18}$.

Proof. We know the full translation complement of the plane $\pi$ and the full stabilizer of a subplane $\rho$. Assume that $h=2^{2 r_{1}+1}$ and $h^{n}=2^{2 r+1}$ so that $n=(2 r+$ $1) /\left(2 r_{1}+1\right)$. The order of the stabilizer subgroup restricted to the line at infinity is

$$
h^{2}\left(h^{2}+1\right)(h-1)(2 r+1) .
$$

Now, in order that a subgroup act transitively on the $h^{2 n}-h^{2}$ components not in $\rho$, we must have

$$
h^{2 n}-h^{2} \text { divides } h^{2}\left(h^{2}+1\right)(h-1)(2 r+1),
$$

so that

$$
h^{2(n-1)}-1 \text { divides }\left(h^{2}+1\right)(h-1)(2 r+1) .
$$

Since $\left(h^{2}+1\right)(h-1)$ divides $h^{4}-1$ and $n-1$ is even, we must have

$$
\frac{h^{2(n-1)}-1}{\left(h^{2}+1\right)(h-1)} \text { divides }(2 r+1), \quad \text { where } h^{n}=2^{2 r+1} \text {. }
$$

Note that $2(n-1)-4>n$ for $n>6$ so that $n$ is 3 or 5 . When $n=5$ then $h=2^{(2 r+1) / 5}$ so that

$$
\frac{2^{8(2 r+1) / 5}-1}{\left(2^{2(2 r+1) / 5}+1\right)\left(2^{(2 r+1) / 5}-1\right)} \text { divides }(2 r+1)
$$

which clearly cannot occur.

Hence, $n=3$.

Now if $n=3$, we must have

$$
2^{(2 r+1) / 3}+1 \text { divides }(2 r+1) .
$$

Thus, it can only be that $2 r+1=3$ or 9 .

It remains to show that we do obtain a cubic extension in either case.

First assume that $2 r+1=9$.

Note that the order of $G_{\rho}$ modulo the kernel is

$$
2^{6}\left(2^{6}+1\right)\left(2^{3}-1\right) 9=2^{6}\left(2^{12}-1\right)=2^{18}-2^{6} .
$$

Thus it remains to show that if an element $g$ of $G_{\rho}$ fixes a component $\ell$ not in $\rho$, then $g \in K^{*}$, where $K^{*}$ denotes the kernel homology group. The stabilizer modulo $K^{*}$ in $G_{\rho}$ of a component $\ell$ has order dividing $\left(2^{9}-1\right) 9$ in the full collineation group. 
Hence, the stabilizer of a component exterior to the components of $\pi_{0}$ by a subgroup of $G_{\rho}$ must have order divisible by $\left(2^{12}-1,\left(2^{9}-1\right) 9\right)=9 \cdot 7$.

Furthermore, since a Sylow 3-group is cyclic, any 3-group must contain an element fixing $\rho$ pointwise. However, if an element of order 3 fixes $\ell$, there would be an element of order 3 fixing a subplane $\rho_{1} \neq \rho$ pointwise.

Thus, the stabilizer of $\ell$ in $G_{\rho}$ (modulo $K^{*}$ ) has order dividing 7. Suppose that an element $g$ of order 7 fixes $\ell$. Then $g$ fixes two components of $\rho$ so cannot be in $S_{z}\left(2^{3}\right)$ as an element of the normalizer of a Sylow 2-subgroup within $S_{z}\left(2^{9}\right)$ fixes exactly two components. Since $g \in S_{z}\left(2^{3}\right) K^{*\left(2^{9}-1\right) /\left(2^{3}-1\right)}$, it follows that $g$ is in $K^{*}$.

Hence, $S_{z}\left(2^{3}\right) C_{9}$ is a group which fixes $\rho$ and acts transitively on the components of $\pi$ and on the components of $\pi-\rho$ so that $\pi$ is a cubic extension of $\rho$.

Now assume that $2 r+1=3$. We notice that the stabilizer subgroup of $\rho$ (now of order 4) is

$$
\left\langle\omega, \tau(a, b), \eta(k) \text {, aut }\left(\rho_{z}\right), s(\alpha)\right\rangle \quad \forall a, b \in K_{0}, \forall k, \alpha \in K_{0}-\{0\}, \text { and } \forall \rho_{z} \in \text { Aut } K \text {. }
$$

This group is still 2-transitive on the set $\Delta$ of infinite points of $\rho$ and has order $4 \cdot 5 \cdot 3$. Since the automorphism group maps

$$
y=x\left[\begin{array}{cc}
b^{\sigma} & b+a^{\sigma+1} \\
b+a^{\sigma+1} & a^{\sigma}
\end{array}\right] \text { onto } y=x\left[\begin{array}{cc}
b^{\tau \sigma} & b^{\tau}+a^{\tau(\sigma+1)} \\
b^{\tau}+a^{\tau(\sigma+1)} & a^{\tau \sigma}
\end{array}\right],
$$

where $\tau$ is an automorphism of $K$, it follows that the collineation group induced from an automorphism group of order 3 is semi-regular on the set $\Gamma$ of infinite points outside of the infinite points of $\rho$. Similarly, it is clear that any group of order 4 is also semi-regular on $\Gamma$. An element of order 5 acting on $2^{6}-2^{2}=60$ components must fix zero or at least five. However, since the group sits in a Suzuki group, it must act fixedpoint-free on $\Gamma$. Hence, the stabilizer subgroup acts transitively on the points of $\Gamma$.

Finally, we note the following corollary.

COROLLARY 2.7. The Lüneburg-Tits plane $\pi$ of order $2^{18}$ is a cubic extension of a Lüneburg-Tits subplane $\pi_{1}$ of order $2^{6}$.

Furthermore, this is the unique cubic extension of a translation plane of order $2^{6}$ with kernel GF $\left(2^{9}\right)$ that admits $S_{z}\left(2^{3}\right)$.

The net of degree $2^{6}+1$ defined by $\pi_{1}$ admits exactly $1+2^{3}+2^{6}$ Lüneburg-Tits subplanes incident with the zero vector.

Proof. To show that the Lüneburg-Tits plane of order $2^{18}$ is the unique cubic extension plane with kernel GF $\left(2^{9}\right)$ that admits $S_{z}\left(2^{3}\right)$, we may appeal to Büttner [2] who uses a computer program to enumerate the spreads in $\mathrm{PG}\left(3,2^{9}\right)$ admitting $S_{z}\left(2^{3}\right)$.

The plane of order $2^{18}$ has kernel GF $\left(2^{9}\right)$ and the subplane has kernel GF $\left(2^{3}\right)$. Hence, there are $\left(2^{9}-1\right) /\left(2^{3}-1\right)=1+2^{3}+2^{6}$ images of a given subplane under the full kernel homology group. We note below that this forces this set to be the full set of subplanes sharing $\Delta$. 
3. Even order cubic extensions. We now point out that only the groups $\operatorname{SL}(2, q)$ or $S_{z}(\sqrt{q})$ are possible for even order cubic extensions. We show initially that the subplanes are always completely determined.

THEOREM 3.1. Let $\pi$ be a cubic extension translation plane of even order $q^{3}$ with subplane $\pi_{0}$ of order $q$. Then $\pi_{0}$ is Desarguesian or Lüneburg-Tits.

Proof. There exists a group of order $q\left(q^{2}-1\right)$ acting on $\pi_{0}$. We assert that the 2-groups must act faithfully. If not there is a Baer involution $\sigma$ with fixed points in $\pi-\pi_{0}$. Hence, there is a subplane of order $q^{3 / 2}$ which contains a subplane of order $q$ which cannot be the case.

First assume that the group is solvable. By Foulser et al. [3, (1.4)], any involution induced on $\pi_{0}$ is not Baer. Hence, an involution must be an elation acting on $\pi_{0}$.

If the group is solvable and an involution in a Sylow 2-subgroup is an elation acting on $\pi_{0}$ then the plane is Desarguesian by Hering [4].

Assume that the group is non-solvable. Since the group is flag-transitive on $\pi_{0}$, we know that $\pi_{0}$ is either Desarguesian or Lüneburg-Tits by Buekenhout et al. [1].

We will show that the involutions are always elations so that the group always contains a more-or-less standard normal subgroup generated by elations. This is accomplished in two theorems. First we establish the nature of the abstract group.

THEOREM 3.2. Let $\pi$ be a translation plane which is a cubic extension of a subplane $\pi_{0}$ of order $q$.

(1) If $q \neq 4$ then the full collineation group $G$ contains a group $H$ acting on $\pi_{0}$ isomorphic to $\operatorname{SL}(2, q)$ or $S_{z}(\sqrt{q})$, respectively, as the subplane $\pi_{0}$ is Desarguesian or Lüneburg-Tits.

(2) If $q=4$ and the group is non-solvable then there is a subgroup acting on $\pi_{0}$ isomorphic to $\mathrm{SL}(2,4)$. If the group is solvable then the Sylow 2-subgroups are cyclic of order 4.

Proof. We know that $\pi_{0}$ is either Desarguesian or Lüneburg-Tits by the previous theorem.

First assume that $\pi_{0}$ is Desarguesian and there is a faithful group induced of order $q$ so that this group is within $\Gamma L(2, q)$. First assume that $q / 2>r$, where $q=2^{r}$. Then, there is a 2 -group in GL $(2, q)$ of order at least 4 which must be an elation group. Since the full group is transitive, the group generated by the elations is also transitive and since there is an elation group of order at least 4 , it follows that $\operatorname{SL}(2, q)$ must be generated. Since the group is transitive on the infinite points of $\pi_{0}$, it follows that the group contains $\operatorname{SL}(2, q)$. If $q / 2 \ngtr r$ then $r=1$ or 2 . Hence, either $\operatorname{SL}(2, q)$ is contained in $G \mid \pi_{0}$ or $q=2$ or 4 .

If $q=2$ then, of course, any translation plane of order $2^{3}$ is Desarguesian but the group is also transitive and the involutions on $\pi_{0}$ are elations and hence $\operatorname{SL}(2,2)$ is generated.

Hence, if we assume that the group is solvable, it must be that $q=2$ or 4 and there are no Baer involutions. 
If $q=4$ assume that there is a 2-group of order at least 8 . Since the induced group lies in $\Gamma L(2,4)$, there are Baer involutions in the solvable case which cannot occur. Hence, the 2-group has order 4 if the group is solvable and is thus, elementary abelian or cyclic. If the group is elementary abelian and there are no Baer involutions, $\mathrm{SL}(2,4)$ is generated. Hence, Baer involutions exist which is a contradiction to solvability. Hence, when the group is solvable, it follows that the 2-groups are cyclic.

Hence, if $q>4$ and $\pi_{0}$ is Desarguesian then the group is nonsolvable and restricted to $\pi_{0}$ contains $\operatorname{SL}(2, q)$.

So, assume that $\pi_{0}$ is a Lüneburg-Tits plane. There are no Baer involutions acting on $\pi_{0}$, so the involutions induce elations on $\pi_{0}$ and each axis is an elation axis. The only involutions acting on $\pi_{0}$ must actually be in $S_{z}(\sqrt{q})$ acting on $\pi_{0}$ since the outer automorphism group has odd order. By Lüneburg [12, (4.12)], the only subgroups of $S_{z}(\sqrt{q})$ which have even order and are transitive are $S_{z}\left(2^{2 m+1}\right)$ type subgroups but again transitivity forces $2^{2 m+1}=\sqrt{q}$.

We now eliminate the possibility that the involutions are Baer.

THEOREM 3.3. Let $\pi$ be a translation plane which is a cubic extension of a subplane $\pi_{0}$ of order $q$.

(1) Then the involutions are elations.

(2) If $q=2^{r}$ and $r$ is odd then $\pi_{0}$ is Desarguesian and the group generated by elations is isomorphic to $\mathrm{SL}(2, q)$.

Proof. Hence, we may assume that $S_{z}(\sqrt{q})$ is induced on the subplane.

We have shown that the 2-groups induce faithfully on $\pi_{0}$ and there is always an elation group of order $\sqrt{q}$ induced on $\pi_{0}$.

Suppose that there is a Baer involution $\sigma$ in the group. Then there is an element of order 2 which fixes a component exterior to the net defined by the components of $\pi_{0}$. However, this means that the Sylow 2-subgroups have order at least $2 q$. Since the 2-groups induce faithfully on $\pi_{0}$, it follows we can only have that $\pi_{0}$ is Desarguesian and $q=2^{r}$, where $r$ is even as the order of a Sylow 2-group divides $q r_{2}$ when $\pi_{0}$ is Desarguesian and divides $q$ when $\pi_{0}$ is Lüneburg-Tits. Furthermore, there must be a Baer involution $\tau_{1}$ inducing a Baer involution on $\pi_{0}$. Hence, if the involutions are not elations then $\pi_{0}$ is Desarguesian. But, note that if the group induced on $\pi_{0}$ is $S_{z}(\sqrt{q})$ then the elations must, in fact, generate a group isomorphic to $S_{z}(\sqrt{q})$.

If the involutions are elations then the group $S_{z}(\sqrt{q})$ occurs only if $r$ is even. We note that all involutions in $S_{z}(\sqrt{q})$ or $\operatorname{SL}(2, q)$ are conjugate.

If involutions inducing elations on $\pi_{0}$ are, in fact, Baer on the plane then $\pi_{0}$ is Desarguesian.

Considering when the subgroup $F$ fixing $\pi_{0}$ pointwise is non-trivial, and $H / F$ is isomorphic to $\mathrm{SL}(2, q)$, the Sylow 2-subgroups are elementary abelian of order $q$.

Now assume that all involutions are Baer. Assume that the full translation complement has Sylow 2-subgroups of order $2^{a} q$. Assume that a Sylow 2-subgroup $S$ contains the subgroup $E$ that induces an elation group of order $q$ on the Desarguesian subplane $\pi_{0}$.

Then, there is a planar subgroup $S^{-}$of order $2^{a}$. Since the group is planar and is 
faithful as acting on the Desarguesian plane $\pi_{0}$, it follows that $S^{-}$is cyclic of order $2^{a}$ and fixes pointwise a Desarguesian subplane $\pi_{0}^{a}$ of order $q^{1 / 2^{a}}$ of $\pi_{0}$. Let $S^{-}=\langle g\rangle$ and let $\sigma$ be in the center of $S$. Let $q=2^{2^{b} z=r}$, for $(2, z)=1$. Notice that acting on $\pi_{0}$, we may represent $S$ as a subgroup of

$$
\left\langle(x, y) \longmapsto\left(x^{2^{z}}, y^{2^{z}}\right), \sigma_{a}:(x, y) \longmapsto(x, x a+y) ; a \in \operatorname{GF}(q)\right\rangle
$$

which has order $2^{b} q$. The center of the latter group consists of the elements $\sigma_{a}$ such that $a^{2^{z}}=a$. Hence, there are involutions in the center of $S$ and such involutions are Baer acting on the plane and are elations acting on the subplane $\pi_{0}$. Hence, $\sigma_{1}$ is in the center of $S$.

First assume that $a>1$. Notice that $g^{2^{a-2}}$ is an involution as acting on Fix $g^{2^{a-1}}$ and $g^{2^{a-1}}$ is a Baer involution leaving Fix $\sigma_{1}$ invariant. We note that $g^{2^{a-1}}$ fixes exactly $\sqrt{q}$ points of the unique component $x=0$ fixed by $S$. Hence, $g^{2^{a-1}}$ cannot be an elation on Fix $\sigma_{1}$ since $(x=0) \cap \pi_{0}$ is contained in a component of Fix $\sigma_{1}$. Hence, $g^{2^{a-1}}$ is a Baer involution on Fix $\sigma_{1}$. That is, Fix $g^{2^{a-1}} \cap$ Fix $\sigma_{1}$ is a subplane of order $q^{3 / 4}$. Since $g^{2^{a-2}}$ is an involution (or trivial) on Fix $g^{2^{a-1}} \cap$ Fix $\sigma_{1}$ and fixes exactly $\sqrt[4]{q}$ points on $x=0$ of $\pi_{0}$, it cannot be an elation (or trivial) on Fix $g^{2^{a-1}} \cap$ Fix $\sigma_{1}$. Hence, $g^{2^{a-2}}$ is a Baer involution on Fix $g^{2^{a-1}} \cap$ Fix $\sigma_{1}$ and fixes exactly a subplane of order $q^{1 / 4}$ of $\pi_{0}$. So, $g^{2^{a-2}}$ fixes a subplane of order $q^{3 / 8}$ of Fix $\sigma_{1}$ so that there are $q^{3 / 8}-q^{1 / 4}$ common fixed components outside of the components of $\pi_{0}$.

Similarly, $g^{2^{a-3}}$ fixes a subplane of order $q^{3 / 16}$ of Fix $\sigma_{1}$ and fixes a subplane of $\pi_{0}$ of order $q^{1 / 8}$ so that there are $q^{3 / 16}-q^{1 / 8}$ common fixed components outside of the components of $\pi_{0}$.

By an easy induction argument, this says that $S^{-}$fixes a subplane pointwise of Fix $\sigma_{1}$ of order $q^{3 / 2^{a+1}}$ and fixes a subplane pointwise of $\pi_{0}$ of order $q^{1 / 2^{a}}$. Hence, there are $q^{3 / 2^{a+1}}-q^{1 / 2^{a}}$ common components fixed by $\sigma_{1}$ and by $S^{-}$. Hence, the stabilizer of one of these common components has order at least $2^{a+1}$, a contradiction.

Now assume that $a=1$. Then $g$ is a Baer involution which induces a Baer involution on $\pi_{0}$ and so Fix $g \cap$ Fix $\sigma_{1}$ is a Baer subplane of Fix $\sigma_{1}$ of order $q^{3 / 4}$ and $g$ fixes exactly $q^{1 / 2}$ points on $\pi_{0} \cap(x=0)$. Thus, there are $q^{3 / 4}-q^{1 / 2}$ common components of Fix $g$ and Fix $\sigma_{1}$ exterior to the components of $\pi_{0}$ which implies that there is a stabilizer 2-group of order at least 4 which is a contradiction. Hence, we have the proof to the theorem.

When $q$ is even, there is a class of translation planes of order $q^{3}$ that admits two groups isomorphic to $\operatorname{GL}(2, q)$ both of which contain a group $\mathscr{Y}$ isomorphic to $\operatorname{SL}(2, q)$ where the involutions are elations. One of these groups is $\mathscr{S} \times K^{*}$, where $K^{*}$ is the kernel homology group of order $q-1$ and $K$ is the kernel of order $q$ which commutes with $\mathscr{Y}$. The other group is defined as follows:

$$
\left\langle\left[\begin{array}{ll}
\alpha & \beta \\
\delta & \gamma
\end{array}\right] \forall \alpha, \beta, \delta, \gamma \in F \ni \alpha \gamma-\beta \delta \neq 0\right\rangle,
$$

where $F$ is a field isomorphic to $K$. The components are $x=0, y=x \alpha$ for all $\alpha$ in $F$ and the images of $y=x T$ under the standard action of the above group where 
$\alpha T=T \alpha^{\sigma}, \sigma$ is an automorphism of $F$. Note that the group elements when $\beta=\sigma=0$ and $\gamma=\alpha^{\sigma}$ define the stabilizer of $y=x T$. We note that the group $K^{*}$ does not leave the subplanes of the elation net invariant whereas the group defined above does leave every subplane invariant.

Hence, it is possible to have a group which is a $\left(q+1, q^{3}-q\right)$-transitive group which leaves a subplane of order $q$ invariant and also a $\left(q+1, q^{3}-q\right)$-transitive group which does not leave a subplane of order $q$ invariant. However, in either case, there is a subplane of order $q$ within the orbit of length $q+1$ and there is a subgroup which leaves the subplane invariant and induces $\operatorname{SL}(2, q)$ on the subplane.

Also, when $\operatorname{SL}(2, q)$ is generated by elations, the elation net is a regulus net and hence there are $1+q+q^{2}$ Desarguesian subplanes incident with the zero vector. In general, the subplane structure is not known when $S_{z}(\sqrt{q})$ acts.

THEOREM 3.4. Let $\pi$ be a translation plane of order $q^{3}$ that admits $S_{z}(\sqrt{q})$ generated by elations.

Then there is a net $\mathcal{N}$ of degree $q+1$ containing either $1,2,3, \sqrt{q}+1$ or $1+\sqrt{q}+q$ Lüneburg-Tits subplanes incident with the zero vector.

Proof. By Hering [5], there is a Lüneburg-Tits subplane invariant under $S_{z}(\sqrt{q})$. Suppose there are three such subplanes sharing the same components say $\pi_{i}$ for $i=1,2,3$ and hence subplanes of $\mathcal{N}$. Let $\mathscr{E}$ denote the enveloping algebra of the net containing the subplanes. Then each subplane $\pi_{i}$ is an irreducible $\mathscr{E}$-module. Hence, if $\pi_{3}$ nontrivially intersects $\pi_{1} \oplus \pi_{2}$, then $\pi_{3}$ lies within the direct sum. We note from Johnson et al. [10] that $\pi_{1} \oplus \pi_{2}$ is a net of degree $1+q$ and order $q^{2}$ and since there are at least three (Baer) subplanes of this net, it follows from Theorem 2.1 that there are $1+\mid$ kernel $\pi_{1} \mid=1+\sqrt{q}$ subplanes of this net. Furthermore, as all of these subplanes are mutually $\mathscr{E}$-isomorphic by the Krull-Schmidt theorem, they are irreducible $\mathscr{E}$-modules and hence, by Liebler [11, Lemma 1.2], it follows that the subplanes of the (sub) net of order $q^{2}$ are also subplanes of the net $\mathcal{N}$.

Now assume that there are three subplanes of the net such that $\pi_{1} \oplus \pi_{2} \oplus \pi_{3}=\pi$. Then assume that there is a fourth subplane $\pi_{4}$ which is not contained in the sum of any two of $\pi_{1}, \pi_{2}$ or $\pi_{3}$. Then, by Theorem 3.1, all subplanes are isomorphic $\mathscr{E}$ submodules and it follows $\mathscr{E}$ is faithful on $\pi_{1}$. Then, there are exactly $1+\sqrt{q}+q$ subplanes of the net $\mathcal{N}$ which are of order $q$ and incident with the zero vector.

THEOREM 3.5. Let $\pi$ be a cubic extension translation plane of even order $q^{3}$ for $q>4$.

Assume that there is a $\left(q+1, q^{3}-q\right)$-transitive group $G$ which does not leave invariant a subplane of order q.

If there is a subplane $\pi_{0}$ of the net of degree $q+1$ such that some subgroup of $G$ leaves $\pi_{0}$ invariant and induces either $\operatorname{SL}(2, q)$ or $S_{z}(\sqrt{q})$ on the subplane then there is a collineation group isomorphic to $\mathrm{SL}(2, q)$ or $S_{z}(\sqrt{q})$ where the involutions are elations.

Furthermore, when $T \simeq \operatorname{SL}(2, q)$ is a collineation group, there are exactly $1+q+q^{2}$ subplanes incident with the zero vector which are left invariant by the group $T$ and when $T \simeq S_{z}(\sqrt{q})$ is a collineation group, there are either $1+\sqrt{q}$ or $1+\sqrt{q}+q$ subplanes which are invariant under $T$. 
Proof. If $\pi_{0}$ of order $q$ admits $\operatorname{SL}(2, q)$ or $S_{z}(\sqrt{q})$, then it follows from the theorem of Lüneburg-Yaqub and Liebler (see Lüneburg [13]) that $\pi_{0}$ is Desarguesian or Lüneburg-Tits and the group acts 2-transitive on the line at infinity of $\pi_{0}$.

Let $S$ be a Sylow 2-subgroup of order $2^{a} q$. The subplanes of order $q$ sharing the components of $\pi_{0}$ are permuted by $S$ and there are $t$ such subplanes where $t \equiv 1 \bmod 2$ such subplanes incident with the zero vector.

Hence, any Sylow 2-subgroup must leave invariant some subplane $\pi_{0}$ on which is also induced a group isomorphic to either $\operatorname{SL}(2, q)$ or $S_{z}(\sqrt{q})$.

The argument of the corresponding previous theorem now applies to finish the result except for the numbers of subplanes.

If $\operatorname{SL}(2, q)$ is a collineation group then since the involutions are elations, the result follows from Ostrom [14].

If $S_{z}(\sqrt{q})$ is a collineation group either there is an invariant subplane or there are at least two subplanes in the net. If there are exactly two, then since the group $S_{z}(\sqrt{q})$ must leave each subplane invariant (as the group is generated by elations), some element $\sigma$ interchanges the two subplanes so that $\sigma^{2}$ fixes both subplanes. Let the order of $\sigma$ be $2^{b} t$, where $(2, t)=1$. If $b=0$, then as $\left\langle\sigma^{2}\right\rangle=\langle\sigma\rangle$, it follows that $\sigma$ fixes both subplanes. Thus, $\sigma^{t}$ has order $2^{b}$ and is not in $S_{z}(\sqrt{q})$ which cannot occur. Hence, there are at least three subplanes provided there are two. Recall that we have a normal subgroup isomorphic to $S_{z}(\sqrt{q})$ and hence a subgroup of order divisible by $(\sqrt{q}+1) /(\sqrt{q}+1, r)$, where $q=2^{r}$ that commutes with $S_{z}(\sqrt{q})$ and thus permutes the subplanes left invariant by $S_{z}(\sqrt{q})$ which are, in fact, all subplanes of the net of degree $q+1$.

Let $\pi_{0}$ and $\pi_{1}$ denote two of the subplanes and consider the subspace $\pi_{0} \oplus \pi_{1}$. Assume that all three subplanes incident with the zero vector are in $\pi_{0} \oplus \pi_{1}$. Then there are exactly $1+\sqrt{q}$ subplanes as the kernel of each subplane is isomorphic to $\mathrm{GF}(\sqrt{q})$. If this is the full set of such subplanes, there is an element $g$ of order dividing $(\sqrt{q}+1) /(\sqrt{q}+1, r)$ which permutes this set of subplanes. If $g$ fixes a subplane $\pi_{1}$ then as the Sylow 2-subgroups fix exactly $\sqrt{q}$ points on each line of $\pi_{1}$, it follows that $g$ fixes $\pi_{1}$ pointwise. But, then $g$ would have to fix an additional subplane pointwise which cannot occur. If not all subplanes are within the direct sum of any two then consider that $\pi_{2}$ is not in $\pi_{0} \oplus \pi_{1}$ so that $\pi=\pi_{0} \oplus \pi_{1} \oplus \pi_{2}$. Since all subplanes are isomorphic and have kernel $\operatorname{GF}(\sqrt{q})$, there is a collineation group of the direct sum isomorphic to $\mathrm{GL}(3, \sqrt{q})$ that fixes each component of the net of degree $q+1$. Furthermore the element $g$ fixes at most one subplane of the net so there are at least $(\sqrt{q}+1) /(\sqrt{q}+$ $1, r)$. We may assume by previous results that $\sqrt{q}>8$, we have $(\sqrt{q}+1) /(\sqrt{q}+1, r)>3$. Thus, the previous result implies that there are exactly $1+\sqrt{q}+\sqrt{q}$ subplanes incident with the zero vector.

4. Solvable extensions. We may complete the problem on solvable extensions for even order as follows.

COROLLARY 4.1. Let $\pi$ be a translation plane of even order $q^{n}$ which is a solvable extension of a flag-transitive plane of order $q$. Then we have one of the following:

(1) $q=2$ or 4 or

(2) $\pi$ is Hall. 
Proof. Apply the main results of Hiramine, Jha and Johnson mentioned in the introduction noting that when $n=3$ and $q>4$ then the group must be non-solvable.

We note that there are examples of solvable $n$-dimensional extensions which are not Hall when $(q, n) \in\{(2,2),(2,3),(2,4),(2,5),(4,3)\}$.

5. Cubic chains. We have noticed that there are chains of cubic extensions. In this section, we indicate the extent of such chains.

THEOREM 5.1. Let $\pi_{0} \subseteq \pi_{1} \subseteq \pi$ be a set of finite translation planes such that $\pi_{1}$ is a cubic extension of $\pi_{0}$ and $\pi$ is a cubic extension of $\pi_{1}$. Assume that the order of $\pi_{0}$ is $q$ so that the orders of $\pi_{1}$ and $\pi$ are $q^{3}$ and $q^{9}$, respectively, where $q$ is even.

Then one of the following occur.

(1) The extensions are nonsolvable-nonsolvable and one of the following occur.

(a) Both $\pi_{0}$ and $\pi_{1}$ are Desarguesian,

(b) $\pi_{0}$ and $\pi_{1}$ are both Lüneburg-Tits planes of orders $q=2^{6}$ and $2^{18}$, respectively.

(2) The extensions are solvable-nonsolvable, $\pi_{0}$ is Desarguesian of order $q=4$ and $\pi_{1}$ is Lüneburg-Tits or Desarguesian of order $4^{3}$.

Proof. By Theorem 3.1, we know that the subplane of a cubic extension is Desarguesian or Lüneburg-Tits.

First assume that the two extensions are, in order, solvable-solvable. Then, by the previous section, if $q>4$ then $\pi_{1}$ is forced to be Hall whereas it is Desarguesian or Lüneburg-Tits by the above remark.

Assume that $q=4$. Then, either both subplanes are Desarguesian or $\pi_{0}$ is Desarguesian and $\pi_{1}$ is Lüneburg-Tits. However, if $\pi_{1}$ is Lüneburg-Tits and $\pi$ is a solvable extension of a plane of order $h=4^{3}>4$ then this forces $\pi$ be to Hall and $\pi_{1}$ to be Desarguesian. Hence, if $q=4$ then both $\pi_{0}$ and $\pi_{1}$ are Desarguesian which forces $\pi$ to be Hall. However, $\pi$ is a cubic extension of a Desarguesian plane $\pi_{1}$ of order $4^{3}$ so the group must contain $\operatorname{SL}\left(2,4^{3}\right)$ which is nonsolvable contrary to the assumption. Hence, $q=4$ does not occur.

If $q=2$ then $\pi_{0}$ and $\pi_{1}$ are Desarguesian of orders 2 and 8 , respectively and solvable-solvable forces $\pi$ to be Hall of order $8^{3}$ which is a contradiction as $8^{3}$ is not square. Hence, solvable-solvable chains do not occur.

Now assume that the extensions are nonsolvable-nonsolvable. If $\pi_{1}$ is LüneburgTits then since a Suzuki group does not contain a nonsolvable SL $\left(2,2^{t}\right)$-subgroup, it follows that $\pi_{0}$ must also be Lüneburg-Tits. By the section on the Lüneburg-Tits planes, it follows that the order $q$ of $\pi_{0}$ must be $2^{6}$ and there are examples here.

Hence, otherwise $\pi_{1}$ must be Desarguesian so that $\pi_{0}$ is forced to be Desarguesian. Examples include the situation when $\pi$ is Desarguesian and the groups are $\operatorname{SL}(2, q) \subseteq$ $\operatorname{SL}\left(2, q^{3}\right)$.

If the extensions are nonsolvable-solvable then since $q^{3}>4$, it follows that $\pi_{1}$ must be Desarguesian and $\pi$ Hall. Hence, $\pi_{0}$ is also Desarguesian. However, we have seen that all involutions of $\pi$ are always elations and that $\operatorname{SL}\left(2, q^{3}\right)$ must always be 
generated from the second extension. Hence, the nonsolvable-solvable situation does not occur.

Now assume that the extensions are solvable-nonsolvable, in order. If $q>4$ again, $\pi_{1}$ is forced to be Hall which cannot be the case. If $q=4$, then $\pi_{0}$ is Desarguesian and $\pi_{1}$ is either Desarguesian or Lüneburg-Tits. If $\pi_{1}$ is Desarguesian then the 2-groups acting on $\pi_{0}$ are cyclic of order 4 and the involutions are elations. Hence, both cases are possible.

\section{REFERENCES}

[1] F. Buekenhout, A. Delandtsheer, J. Doyen, P. B. Kleidman, M. W. Liebeck, and J. Saxl, Linear spaces with flag-transitive automorphism groups, Geometriae Dedicata 36 (1990), no. 1, 89-94. MR 91j:20009. Zbl 707.51017.

[2] W. Büttner, Darstellungstheoretische Methoden zur Konstruktion Endlicher Translationsebenen der Charakteristik, Habitationschrift, Fachbereich Math. Technischen Hochschule Darmstadt, 1983.

[3] D. A. Foulser and M. J. Kallaher, Solvable, flag-transitive, rank 3 collineation groups, Geometriae Dedicata 7 (1978), no. 1, 111-130. MR 57\#12263. Zbl 406.51009.

[4] C. Hering, On finite line transitive affine planes, Geometriae Dedicata 1 (1973), no. 4, 387-398. MR 48\#12275. Zbl 271.50002.

[5] _ On projective planes of type VI, Colloquio Internazionale sulle Teorie Combinatorie (Rome, 1973), Tomo II, Atti dei Convegni Lincei, no. 17, Accad. Naz. Lincei, Rome, 1976, pp. 29-53. MR 57\#7367. Zbl 355.50010.

[6] Y. Hiramine, V. Jha, and N. L. Johnson, Solvable extensions of flag-transitive planes, to appear in Note Mat.

[7] _ Quadratic extensions of flag-transitive planes, European J. Combin. 20 (1999), no. 8, 797-818. MR 2000k:51002. Zbl 991.26181.

[8] V. Jha and N. L. Johnson, An analog of the Albert-Knuth theorem on the orders of finite semifields, and a complete solution of Cofman's subplane problem, Algebras Groups Geom. 6 (1989), no. 1, 1-35. MR 90i:51009. Zbl 684.51003.

[9] _ A geometric characterization of generalized Desarguesian planes, Atti Sem. Mat. Fis. Univ. Modena 38 (1990), no. 1, 71-80. MR 92f:51007. Zbl 708.51004.

[10] N. L. Johnson and T. G. Ostrom, Direct products of affine partial linear spaces, J. Combin. Theory Ser. A 75 (1996), no. 1, 99-140. MR 97f:51014. Zbl 873.51001.

[11] R. A. Liebler, Combinatorial representation theory and translation planes, Finite Geometries (Pullman, Wash., 1981), Lecture Notes in Pure and Appl. Math., vol. 82, Dekker, New York, 1983, pp. 307-325. MR 85g:51007. Zbl 574.51015.

[12] H. Lüneburg, Die Suzukigruppen und ihre Geometrien. Vorlesung Sommersemester 1965 in Mainz, Springer-Verlag, Berlin, 1965 (German). MR 34\#7634. Zbl 136.01502.

[13] _ Translation Planes, Springer-Verlag, Berlin, 1980. MR 83h:51008. Zbl 446.51003.

[14] T. G. Ostrom, Linear transformations and collineations of translation planes, J. Algebra 14 (1970), 405-416. MR 41\#6049. Zbl 188.24403.

Yutaka Hiramine: Department of Mathematics, Faculty of Education, Kumamoto UNIVERSITY, KUROKAMI, KUMAMOTO, JAPAN

E-mail address: hi ramine@gpo.kumamoto-u.ac.jp

Vikram Jha: Mathematics Department, Caledonian University, CoWCaddens Road, GLASGOW, SCOTLAND

E-mail address: v.jha@gca1.ac.uk

NORMAN L. JOHNSON: UNIVERSITY OF IOWA, IOWA CITY, IA 52242, USA

E-mail address: njohnson@math . ui owa . edu 


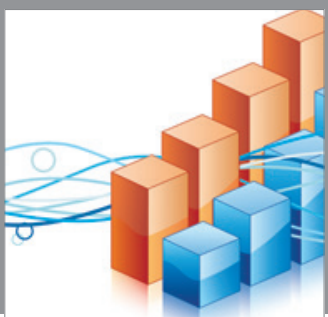

Advances in

Operations Research

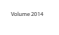

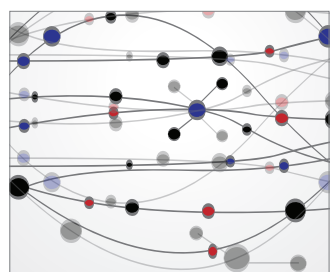

\section{The Scientific} World Journal
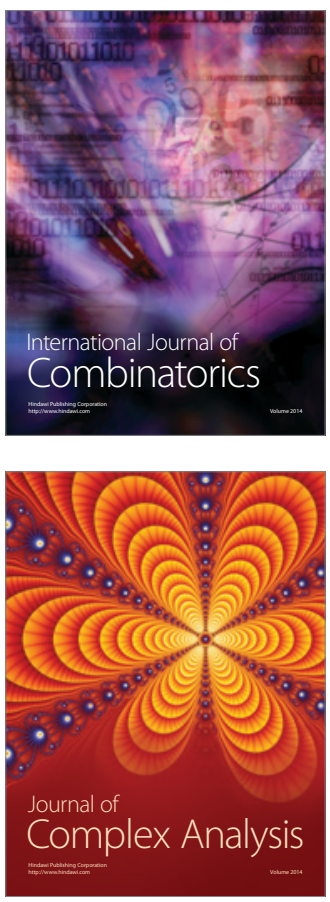

International Journal of

Mathematics and

Mathematical

Sciences
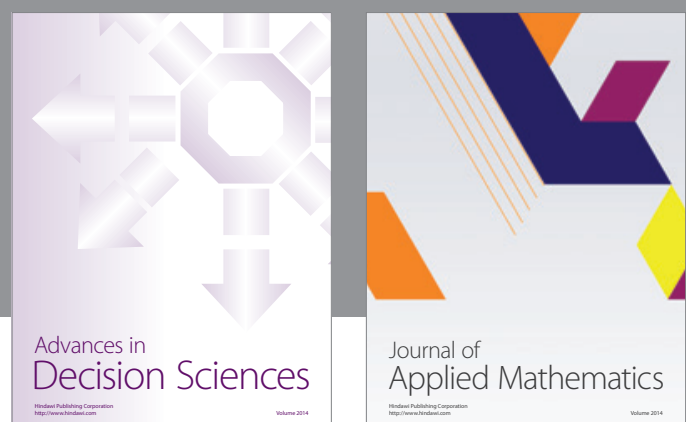

Journal of

Applied Mathematics
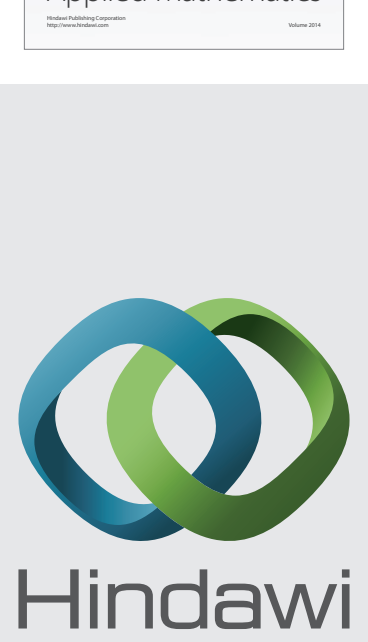

Submit your manuscripts at http://www.hindawi.com
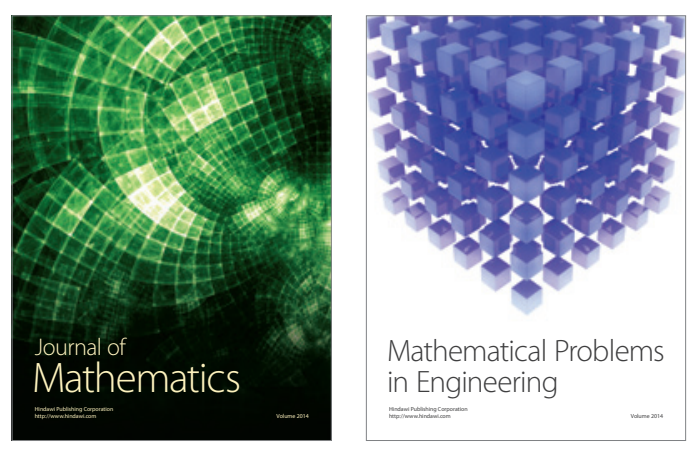

Mathematical Problems in Engineering
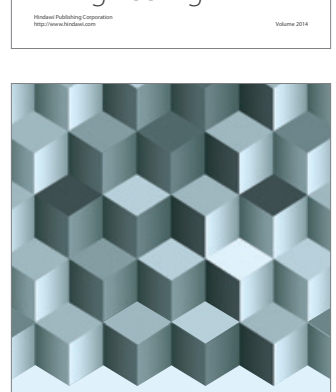

Journal of

Function Spaces
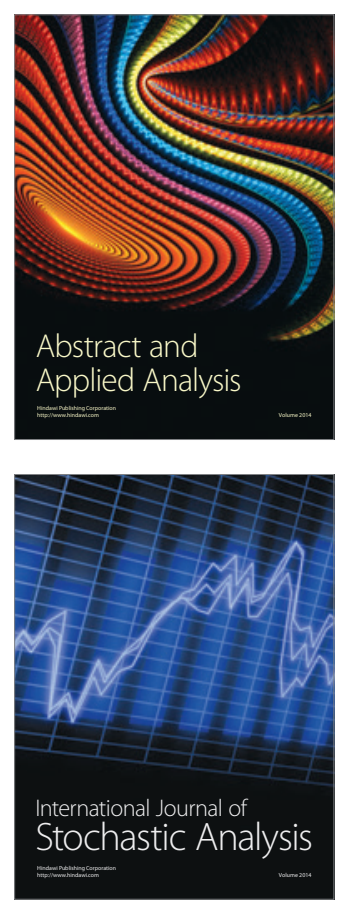

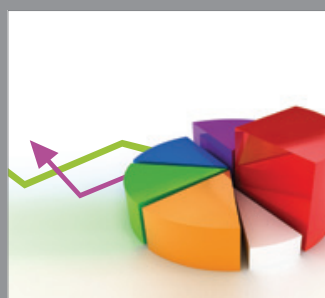

ournal of

Probability and Statistics

Promensencen
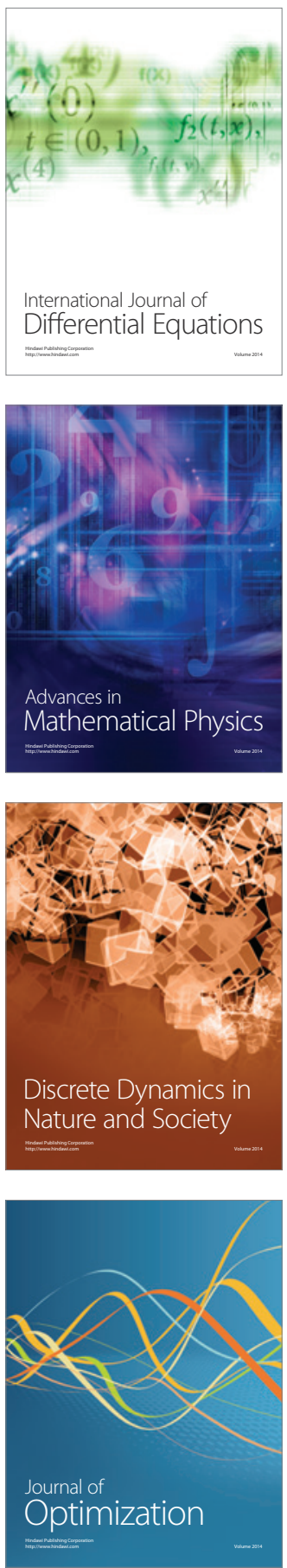